\title{
Textbook outcome and survival of robotic versus laparoscopic total gastrectomy for gastric cancer: A propensity score matched cohort study
}

\section{Chul Kyu Roh}

Ajou University School of Medicine

\section{Soomin Lee}

Ajou University School of Medicine

\section{Sang-Yong Son}

Ajou University School of Medicine

Hoon Hur

Ajou University School of Medicine

Sang-Uk Han ( $\sim$ hansu@ajou.ac.kr)

Ajou University School of Medicine

\section{Research Article}

Keywords: gastrectomy, gastric cancer, textbook outcome, survival outcome

Posted Date: May 27th, 2021

DOI: https://doi.org/10.21203/rs.3.rs-560188/v1

License: @ (i) This work is licensed under a Creative Commons Attribution 4.0 International License. Read Full License

Version of Record: A version of this preprint was published at Scientific Reports on July 28th, 2021. See the published version at https://doi.org/10.1038/s41598-021-95017-3. 


\section{Abstract}

Introduction Textbook outcome is a composite quality measurement of short-term outcomes for evaluating complex surgical procedures. We compared textbook outcome and survival of robotic total gastrectomy(RTG) with those of laparoscopic total gastrectomy(LTG).

Methods We retrospectively reviewed 395patients(RTG,n=74;LTG, $n=321$ ) who underwent curative total gastrectomy for gastric cancer via minimally invasive approaches from 2009 to 2018. We performed propensity score matched analysis to adjust for potential selection bias. Textbook outcome included a negative resection margin, no intraoperative complication, retrieved lymph nodes $>15$, no severe complication, no reintervention, no unplanned intensive care unit admission, hospitalisation $\leq 21$ days, no readmission after discharge, and no postoperative mortality. Survival outcomes included 5-year overall and relapse-free survival rates.

Results After matching, 74patients in each group were selected. Textbook outcome was similar in the RTG and LTG groups(70.3\% and 75.7\%,respectively), although RTG required a longer operative time. The quality metric least often achieved was the presence of severe complications in both groups( $77.0 \%$ in both groups). There were no differences in the 5-year overall survival rate( $90.7 \%$ and $85.9 \%$,respectively;log-rank $P=0.144)$ and relapse-free survival rate between the RTG and LTG groups(89.5\% and $85.2 \%$,respectively;log-rank $P=0.167$ ).

Conclusion Textbook outcome and survival outcome of RTG were comparable to those of LTG for gastric cancer.

\section{Introduction}

Quality assurance in surgical oncology is an important factor in evaluating the outcomes of surgical procedures[1, 2]. Traditionally, quality assessment for gastrectomy has focused on surgical mortality and morbidity[3-5]. Since it only provides information on single-quality indicators, it does not fully measure the complex aspects of perioperative surgical care in gastric cancer.

Investigators have been increasingly searching for standards for perioperative outcomes. In 2017, the Dutch Upper Gastrointestinal Cancer Audit group developed a composite measure defined as textbook outcome that combined 10 outcome parameters to assess the quality of surgical care for gastric cancer[6]. Several studies have reported that textbook outcome can be used as the standard for optimisation of care and is associated with long-term survival in patients with gastric cancer[7-10]. Additionally, textbook outcome has been applied to comparisons of quality across surgical practices and institutions in various fields, such as colon, oesophagus, liver, and pancreatic cancers[7, 11-13].

Recently, robotic gastrectomy showed reliable surgical outcomes as an alternative for laparoscopic gastrectomy in minimally invasive surgery for the treatment of gastric cancer[14-19]. However, these studies focused on singlequality indicators such as bleeding, retrieved lymph nodes, complications, and mortality to compare short-term outcomes. Unfortunately, no studies have yet utilised textbook outcome to compare short-term surgical outcomes between robotic and laparoscopic gastrectomy. Further, there have been few studies that compare survival outcomes of robotic total gastrectomy (RTG) with those of laparoscopic total gastrectomy (LTG).

Therefore, we aimed to compare the perioperative (based on textbook outcome) and survival outcomes between RTG and LTG for gastric cancer treatment. 


\section{Methods}

\section{Patients}

We reviewed the prospectively collected data of patients who underwent minimally invasive (robotic or laparoscopic) total gastrectomy for gastric cancer from January 2009 to December 2018 at the Department of Surgery, Ajou University School of Medicine. All gastrectomy cases were performed by three surgeons (HSU, HH, and SSY). The exclusion criteria were as follows: palliative gastrectomy, emergency surgery, and remnant gastric cancer that required completion total gastrectomy. Patients were given the option of undergoing robotic or laparoscopic gastrectomy and each patient selected the type of surgery. Written informed consent was provided prior to surgery. The institutional review board of Ajou University Hospital, Suwon, Korea approved this study (approval number: AJIRB-MED-MDB-19-144).

\section{Surgical procedures}

\section{Robotic total gastrectomy}

RTG was first initiated at our institution in January 2009. We performed RTG using the da Vinci® S, Si, or Xi Systems (Intuitive Surgical, Sunnyvale, CA, USA). Typical surgical procedures for RTG have been previously described in detail[20]. We performed D1 + or D2 lymph node dissection (LND) based on the Korean and Japanese gastric cancer treatment guidelines[21, 22]. Esophagojejunostomy was performed extracorporeally using circular staplers, or intracorporeally using linear staplers or by suture technique. Until 2014, a mini laparotomy was created in the upper midline (approximately $7 \mathrm{~cm}$ ) after oesophageal mobilisation. Esophagojejunostomy was performed using a 25-mm or 21-mm circular stapler, and jejunojejunostomy was performed. Since 2015, esophagojejunostomy and jejunojejunostomy were performed intracorporeally using linear staplers. A 45-mm linear stapler was inserted individually between the oesophagus and prepared Roux limb, and fired to form a sideto-side esophagojejunostomy. Suture technique involved an intracorporeally robot-sewn esophagojejunostomy. After transection of the oesophagus, seromuscular sutures were performed to attach the oesophageal stump and jejunum together.

\section{Laparoscopic total gastrectomy}

We performed LTG with lymphadenectomy based on standardised operative strategies, as described previously[20]. The extent of LND was decided according to the Korean and Japanese gastric cancer treatment guidelines[21, 22]. The reconstruction method was performed by extracorporeal or intracorporeal anastomosis as completed in RTG.

\section{Textbook outcome}

Textbook outcome is a composite of nine components involved in the perioperative process, including the oncologic resection, postoperative care, and discharge of gastric cancer patients. We excluded the intent of surgery, which was one of the parameters of textbook outcome, from the definition to reduce selection bias for survival analysis in this study.

Textbook outcome was defined as the percentage of patients who had (a) no intraoperative complication, (b) tumour-negative resection margins, (c) $>15$ lymph nodes retrieved, (d) no severe postoperative complication (severe complications were defined as grade II or more complications according to the Clavien-Dindo 
classification), (e) no re-intervention (endoscopic or radiological re-intervention was not considered), (f) no

unplanned admission to the intensive care unit, $(\mathrm{g})$ hospitalisation period of $\leq 21$ days, (h) no postoperative mortality within 30 days after surgery, and (i) no readmission after discharge. Textbook outcome was achieved when all these metrics were met.

\section{Follow-up}

Follow-up data after surgery of all patients were collected from our institution's database. Patients with stage I cancer were regularly surveyed according to standard protocol (i.e., at 3-month intervals for the first year, 6-month intervals for the next 4 years, and yearly thereafter). Patients with stage II cancer or higher were surveyed every three months for the first two years, every six months for the next three years, and yearly thereafter. Physical examination, laboratory testing, esophagogastroduodenoscopy, and imaging (ultrasonography or computed tomography) were conducted during the follow-up. Recurrence was assessed by radiology, endoscopy, surgery, or clinical signs of the disease.

5-Fluorouracil-based adjuvant chemotherapy (e.g., S-1 monotherapy or oxaliplatin with capecitabine) was suggested to patients with pathological stage II or III cancer. Survival status for all patients was confirmed. Allcause and cancer mortality data were acquired from the Korean National Cancer Registry. Patient follow-up was completed until death or the last follow-up for all patients (data cut-off: December 31, 2019).

\section{Propensity score matching}

Propensity score matching analysis was performed to adjust for potential selection bias between the groups. Among clinicopathological variables, the covariates with a standardised mean difference (SMD) $>0.1$ in the entire cohort were selected for matching to balance significant differences in baseline characteristics as follows: age, sex, body mass index (BMI), the American Society of Anesthesiologist (ASA) physical status, tumour size, and the pathological stage of gastric cancer. TNM stage was based on the 8th edition of the American Joint Committee on Cancer staging for gastric cancer. Individual propensity scores were calculated using a logistic regression model, and patients between the two groups were matched using the nearest-neighbour matching algorithm (ratio $=1: 1$ without replacement) with a caliper width of 0.25 standard deviation of the propensity score.

\section{Statistical analyses}

Data analyses were performed using IBM SPSS Statistics for Windows, version 25.0 (IBM Corp., Armonk, NY, USA). Continuous variables were analysed using the Student's t-test to compare mean values. Categorical variables, which are presented as numbers (n) and percentages (\%), were analysed using the chi-square test or Fisher's exact test. Overall survival was defined as the time of gastrectomy to the time of all-cause death. Relapse-free survival was defined as the time of gastrectomy to the time of tumour recurrence or all-cause death. Survival curves were estimated using the Kaplan-Meier method for the entire cohort and for the matched cohorts. To investigate prognostic variables associated with overall and relapse-free survival, Cox proportional hazard regression model was used for univariate and multivariate analysis. Statistical significance was defined as a $P<0.05$.

\section{Results}

\section{Study cohort}


Four hundred and thirty-four patients who underwent gastrectomy from 2009 to 2018 for gastric cancer by either robot or laparoscopy were identified. Of these, 39 patients were excluded who underwent palliative gastrectomy (n $=28$ ) or completion total gastrectomy for remnant gastric cancer $(n=10)$, or emergency surgery due to cancer bleeding $(n=1)$. Finally, 395 patients (RTG, $n=74 ;$ LTG, $n=321)$ were included. After matching, 148 patients (74 patients per group) were analysed to compare textbook and survival outcomes between the two groups.

\section{Patient characteristics}

Before matching, the mean age of the RTG group ( $53.8 \pm 11.6$ years) was lower than that of the LTG group (60.7 \pm 12.6 years; $P<0.001, \mathrm{SMD}=0.590)$. The RTG group $(43.2 \%)$ exhibited a higher proportion of female sex than the LTG group $(30.5 \% ; P=0.040, \mathrm{SMD}=0.255)$. The proportion of patients with ASA class I was higher in the RTG group than in the LTG group $(67.6 \%$ versus [vs.] $47.0 \% ; P=0.007, \mathrm{SMD}=0.380)$. After propensity score matching, clinicopathological variables were well balanced within the SMD of 0.1 between the two groups (Table 1). 
Table 1

Clinicopathologic characteristics before and after propensity score matching

\begin{tabular}{|c|c|c|c|c|c|c|c|c|}
\hline & \multicolumn{4}{|c|}{ Entire cohort } & \multicolumn{4}{|c|}{ Matched cohort } \\
\hline & $\begin{array}{l}\text { Robot } \\
(n= \\
74)\end{array}$ & $\begin{array}{l}\text { Laparoscopy } \\
(n=321)\end{array}$ & $\mathbf{P}$ & SMD & $\begin{array}{l}\text { Robot } \\
(n= \\
74)\end{array}$ & $\begin{array}{l}\text { Laparoscopy } \\
(n=74)\end{array}$ & $\mathbf{P}$ & SMD \\
\hline Age, years & $\begin{array}{l}53.8 \pm \\
11.6\end{array}$ & $60.7 \pm 12.6$ & $\begin{array}{l}< \\
0.001\end{array}$ & 0.590 & $\begin{array}{l}53.8 \pm \\
11.6\end{array}$ & $54.6 \pm 12.7$ & 0.690 & 0.069 \\
\hline Sex & & & 0.040 & 0.255 & & & $\vec{D}_{0.999}$ & $\begin{array}{l}< \\
0.001\end{array}$ \\
\hline Male & $\begin{array}{l}42 \\
(56.8)\end{array}$ & $223(69.5)$ & & & $\begin{array}{l}42 \\
(56.8)\end{array}$ & $42(56.8)$ & & \\
\hline Female & $\begin{array}{l}32 \\
(43.2)\end{array}$ & $98(30.5)$ & & & $\begin{array}{l}32 \\
(43.2)\end{array}$ & $32(43.2)$ & & \\
\hline $\begin{array}{l}\text { Body mass index, } \\
\mathrm{kg} / \mathrm{m}^{2}\end{array}$ & $\begin{array}{l}23.6 \pm \\
2.9\end{array}$ & $24.0 \pm 3.2$ & 0.262 & 0.154 & $\begin{array}{l}23.6 \pm \\
2.9\end{array}$ & $23.8 \pm 3.4$ & 0.692 & 0.071 \\
\hline ASA group & & & 0.007 & 0.380 & & & $\overrightarrow{ }_{0.999}$ & 0.051 \\
\hline I & $\begin{array}{l}50 \\
(67.6)\end{array}$ & $151(47.0)$ & & & $\begin{array}{l}50 \\
(67.6)\end{array}$ & $51(68.9)$ & & \\
\hline II & $\begin{array}{l}22 \\
(29.7)\end{array}$ & $162(50.5)$ & & & $\begin{array}{l}22 \\
(29.7)\end{array}$ & $22(29.7)$ & & \\
\hline III & $2(2.7)$ & $8(2.5)$ & & & $2(2.7)$ & $1(1.4)$ & & \\
\hline Tumor histology & & & 0.238 & 0.160 & & & $\vec{D}_{0.999}$ & 0.027 \\
\hline Differentiated & $\begin{array}{l}39 \\
(52.7)\end{array}$ & $195(60.7)$ & & & $\begin{array}{l}39 \\
(52.7)\end{array}$ & $38(51.4)$ & & \\
\hline Undifferentiated & $\begin{array}{l}35 \\
(47.3)\end{array}$ & $126(39.3)$ & & & $\begin{array}{l}35 \\
(47.3)\end{array}$ & $36(48.6)$ & & \\
\hline Tumor location & & & $\overrightarrow{ }_{0.999}$ & 0.072 & & & 0.723 & 0.116 \\
\hline Upper third & $\begin{array}{l}51 \\
(68.9)\end{array}$ & $221(68.9)$ & & & $\begin{array}{l}51 \\
(68.9)\end{array}$ & $47(63.5)$ & & \\
\hline Middle to lower third & $\begin{array}{l}23 \\
(31.1)\end{array}$ & $100(32.2)$ & & & $\begin{array}{l}23 \\
(31.1)\end{array}$ & $27(36.5)$ & & \\
\hline
\end{tabular}

Data are expressed as mean \pm standard deviations or as $n(\%)$ unless otherwise specified.

*According to American Joint Committee on Cancer 8th edition.

†For patients with Stage II or III

SMD, standardized mean difference; ASA, American Society of Anaesthesiologists 


\begin{tabular}{|c|c|c|c|c|c|c|c|c|}
\hline & Entire c & lort & & & Matche & cohort & & \\
\hline & $\begin{array}{l}\text { Robot } \\
(n= \\
74)\end{array}$ & $\begin{array}{l}\text { Laparoscopy } \\
(n=321)\end{array}$ & $\mathbf{P}$ & SMD & $\begin{array}{l}\text { Robot } \\
(n= \\
74)\end{array}$ & $\begin{array}{l}\text { Laparoscopy } \\
(n=74)\end{array}$ & $\mathbf{P}$ & SMD \\
\hline $\begin{array}{l}\text { Clinical T } \\
\text { classification }\end{array}$ & & & 1.000 & 0.116 & & & 0.869 & 0.054 \\
\hline T1 & $\begin{array}{l}39 \\
(52.7)\end{array}$ & $167(52.0)$ & & & $\begin{array}{l}39 \\
(52.7)\end{array}$ & $37(50.0)$ & & \\
\hline$\geq \mathrm{T} 2$ & $\begin{array}{l}35 \\
(47.3)\end{array}$ & $154(48.0)$ & & & $\begin{array}{l}35 \\
(47.3)\end{array}$ & $37(50.0)$ & & \\
\hline $\begin{array}{l}\text { Clinical N } \\
\text { classification }\end{array}$ & & & 1.000 & 0.057 & & & $\begin{array}{l}> \\
0.999\end{array}$ & 0.029 \\
\hline No & $\begin{array}{l}50 \\
(67.6)\end{array}$ & $216(67.3)$ & & & $\begin{array}{l}50 \\
(67.6)\end{array}$ & $49(66.2)$ & & \\
\hline$\geq \mathrm{N} 1$ & $\begin{array}{l}24 \\
(32.4)\end{array}$ & 105 (32.7) & & & $\begin{array}{l}24 \\
(32.4)\end{array}$ & $25(33.8)$ & & \\
\hline Tumor size, $\mathrm{cm}$ & $\begin{array}{l}3.5 \pm \\
1.7\end{array}$ & $4.0 \pm 2.9$ & 0.078 & 0.294 & $\begin{array}{l}3.5 \pm \\
1.7\end{array}$ & $3.5 \pm 2.6$ & 0.982 & 0.005 \\
\hline $\begin{array}{l}\text { Pathological T } \\
\text { classification }\end{array}$ & & & 0.813 & 0.053 & & & 0.314 & 0.013 \\
\hline T1 & $\begin{array}{l}43 \\
(58.1)\end{array}$ & $184(57.3)$ & & & $\begin{array}{l}43 \\
(58.1)\end{array}$ & $43(58.1)$ & & \\
\hline T2 & $\begin{array}{l}12 \\
(16.2)\end{array}$ & $41(12.8)$ & & & $\begin{array}{l}12 \\
(16.2)\end{array}$ & $9(12.2)$ & & \\
\hline T3 & $\begin{array}{l}12 \\
(16.2)\end{array}$ & $64(19.9)$ & & & $\begin{array}{l}12 \\
(16.2)\end{array}$ & 19 (25.7) & & \\
\hline $\mathrm{T} 4$ & $7(9.5)$ & $32(10.0)$ & & & $7(9.5)$ & $3(4.1)$ & & \\
\hline $\begin{array}{l}\text { Pathological N } \\
\text { classification }\end{array}$ & & & 0.592 & 0.134 & & & 0.382 & 0.091 \\
\hline NO & $\begin{array}{l}53 \\
(71.6)\end{array}$ & $215(67.0)$ & & & $\begin{array}{l}53 \\
(71.6)\end{array}$ & $46(62.2)$ & & \\
\hline N1 & $\begin{array}{l}9 \\
(12.2)\end{array}$ & $45(14.0)$ & & & $\begin{array}{l}9 \\
(12.2)\end{array}$ & $17(23.0)$ & & \\
\hline N2 & $\begin{array}{l}8 \\
(10.8)\end{array}$ & $29(9.0)$ & & & $\begin{array}{l}8 \\
(10.8)\end{array}$ & $7(9.5)$ & & \\
\hline
\end{tabular}

Data are expressed as mean \pm standard deviations or as $n(\%)$ unless otherwise specified.

*According to American Joint Committee on Cancer 8th edition.

†For patients with Stage II or III

SMD, standardized mean difference; ASA, American Society of Anaesthesiologists 


\begin{tabular}{|c|c|c|c|c|c|c|c|c|}
\hline & \multicolumn{4}{|c|}{ Entire cohort } & \multicolumn{4}{|c|}{ Matched cohort } \\
\hline & $\begin{array}{l}\text { Robot } \\
(n= \\
74)\end{array}$ & $\begin{array}{l}\text { Laparoscopy } \\
(n=321)\end{array}$ & $P$ & SMD & $\begin{array}{l}\text { Robot } \\
(n= \\
74)\end{array}$ & $\begin{array}{l}\text { Laparoscopy } \\
(n=74)\end{array}$ & $P$ & SMD \\
\hline N3 & $4(5.4)$ & $32(10.0)$ & & & $4(5.4)$ & $4(5.4)$ & & \\
\hline $\begin{array}{l}\text { Pathological TNM } \\
\text { classification* }\end{array}$ & & & 0.320 & 0.145 & & & 0.829 & 0.058 \\
\hline I & $\begin{array}{l}50 \\
(67.6)\end{array}$ & 207 (64.5) & & & $\begin{array}{l}50 \\
(67.6)\end{array}$ & $47(63.5)$ & & \\
\hline II & $\begin{array}{l}15 \\
(20.3)\end{array}$ & $52(16.2)$ & & & $\begin{array}{l}15 \\
(20.3)\end{array}$ & 18 (24.3) & & \\
\hline III & $\begin{array}{l}9 \\
(12.2)\end{array}$ & 62 (19.3) & & & $\begin{array}{l}9 \\
(12.2)\end{array}$ & $9(12.2)$ & & \\
\hline $\begin{array}{l}\text { Adjuvant } \\
\text { chemotherapy } \dagger\end{array}$ & & & $>_{0.999}$ & 0.065 & & & $\overrightarrow{0.999}$ & 0.086 \\
\hline Yes & $\begin{array}{l}22 \\
(91.7)\end{array}$ & $105(92.1)$ & & & $\begin{array}{l}22 \\
(91.7)\end{array}$ & 25 (92.6) & & \\
\hline No & $2(8.3)$ & $9(7.9)$ & & & $2(8.3)$ & $2(7.4)$ & & \\
\hline $\begin{array}{l}\text { Chronological } \\
\text { distribution }\end{array}$ & & & 0.115 & 0.205 & & & 0.869 & 0.054 \\
\hline $2009-2013$ & $\begin{array}{l}36 \\
(48.6)\end{array}$ & $123(38.3)$ & & & $\begin{array}{l}36 \\
(48.6)\end{array}$ & $34(45.9)$ & & \\
\hline 2014-2018 & $\begin{array}{l}38 \\
(51.4)\end{array}$ & $198(61.7)$ & & & $\begin{array}{l}38 \\
(51.4)\end{array}$ & $40(54.1)$ & & \\
\hline \multicolumn{9}{|c|}{ Data are expressed as mean \pm standard deviations or as $n(\%)$ unless otherwise specified. } \\
\hline \multicolumn{9}{|c|}{ *According to American Joint Committee on Cancer 8th edition. } \\
\hline \multicolumn{9}{|c|}{ †For patients with Stage II or III } \\
\hline
\end{tabular}

\section{Operative outcomes}

In the matched cohort, a longer operative time was required in the RTG group than in the LTG group $(226.0 \pm 56.9$ vs. $193.2 \pm 46.8 \mathrm{~min} ; P<0.001, \mathrm{SMD}=0.578$ ). The estimated blood loss tended to be less in the RTG group than in the LTG group, although this was not significantly different. The esophagojejunostomy method was comparable between the groups. No conversion to laparoscopic or open surgery occurred in the RTG group; however, at any time during the operation, the surgeon had the option of conversion to conventional laparoscopy or open surgery for patients' safety. Perioperative outcomes regarding the extent of dissection, number of retrieved lymph nodes, tumour-free resection margins, time to soft diet, and duration of hospital stay were comparable in both groups. 
The incidence and grade of complications were also similar in both groups. We did not experience any mortality within the first 30 days after total gastrectomy by either robot or laparoscopy (Table 2). 
Table 2

Operative outcomes before and after propensity score matching

Entire cohort

$\begin{array}{ll}\text { Robot } & \text { Laparoscop } \\ (n= & (n=321) \\ 74) & \end{array}$
Matched cohort

$\begin{array}{lll}\text { SMD } & \text { Robot } & \text { Laparosco } \\ & (n= & (n=74) \\ & 74) & \end{array}$

Extent of dissection

$<$ D2

$\geq \mathrm{D} 2$

Esophagojejunostomy method

Circular stapler

37
$(50.0)$

Linear stapler

34

(45.9)

36
$(48.6)$

38

(51.4)

$171(53.3)$

$150(46.7)$

$0.519 \quad 0.092$

$\begin{array}{ll}36 & 34(45.9)\end{array}$

(48.6)

38

(51.4) 40 (54.1)

$\begin{array}{llll}0.010 & 0.084 & & \\ & & & \\ & 37 & 41(55.4) \\ & (50.0) & \\ & 34 & 33(44.6) \\ & (45.9) & \end{array}$

$163(50.8)$

158 (49.2)

$0.869 \quad 0.054$

SMD

Suture technique

3

$0(0)$

3
$(4.1)$

Combined resection 5

$0(0)$

Conversion*

$28(8.7)$

$0.651 \quad 0.078$

5
$(6.8)$

4 (5.4)

0.283

0.164

$0(0)$

$4(1.2)$

0.595 NA

$0(0)$

$1(1.4)$

0.999

0.053

Operation time, min

Estimated blood loss,

$\mathrm{ml}$

\begin{tabular}{ll}
226.0 & $191.7 \pm$ \\
\pm 56.9 & \\
\hline 137.9 & $153.6 \pm$ \\
\pm & 150.0 \\
155.5 &
\end{tabular}

Proximal margin, cm

$\begin{array}{ll}2.5 \pm & 2.8 \pm 2.3 \\ 1.5 & \end{array}$

Distal margin, cm

$$
\begin{aligned}
& 11.9 \\
& \pm 3.4
\end{aligned}
$$

$11.7 \pm 4.2$

Retrieved LNs, $n$

$$
\begin{aligned}
& 43.1 \\
& \pm 14.9
\end{aligned}
$$

$42.9 \pm 15.0$

0.916

0.278

0.158

0.604

226.

226.0
\pm 56.9

$193.2 \pm 46.8$

> 0.999

NA

Time to start soft diet, days
$6.1 \pm$
$5.7 \pm 2.8$
4.0

$0.377 \quad 0.109$

$\begin{array}{ll}137.9 & 149.1 \pm \\ \pm & 128.3 \\ 155.5 & \end{array}$

0.001

0.578

0.420

0.101

$\pm$

$0.632 \quad 0.072$

$\begin{array}{lll}2.5 \pm & 2.8 \pm 2.0 & 0.341\end{array}$

0.183

$0.644 \quad 0.063 \quad 11.9$

11.9
\pm 3.4

$11.9 \pm 4.0$

$0.989 \quad 0.002$

43.1

$46.0 \pm 14.4$

0.232

0.194 $\pm 14.9$

$\begin{array}{llll}6.1 \pm & 6.4 \pm 4.8 & 0.670 & 0.078 \\ 4.0 & & & \end{array}$

Data are expressed as means \pm standard deviations or as $n(\%)$ unless otherwise specified.

*Conversion to laparoscopic or open surgery in the robotic group and conversion to open surgery in the laparoscopic group for any reason.

SMD, standardized mean difference; LNs, lymph nodes; NA, not applicable. 


\begin{tabular}{|c|c|c|c|c|c|c|c|c|}
\hline & \multicolumn{4}{|c|}{ Entire cohort } & \multicolumn{4}{|c|}{ Matched cohort } \\
\hline & $\begin{array}{l}\text { Robot } \\
(n= \\
74)\end{array}$ & $\begin{array}{l}\text { Laparoscopy } \\
(n=321)\end{array}$ & $\mathbf{P}$ & SMD & $\begin{array}{l}\text { Robot } \\
(n= \\
74)\end{array}$ & $\begin{array}{l}\text { Laparoscopy } \\
(n=74)\end{array}$ & $\mathbf{P}$ & SMD \\
\hline $\begin{array}{l}\text { Postoperative hospital } \\
\text { stay, days }\end{array}$ & $\begin{array}{l}9.0 \pm \\
5.0\end{array}$ & $8.2 \pm 4.4$ & 0.214 & 0.146 & $\begin{array}{l}9.0 \pm \\
5.0\end{array}$ & $9.2 \pm 5.7$ & 0.795 & 0.046 \\
\hline Complications & & & 0.304 & 0.132 & & & $\overrightarrow{0.999}$ & $\begin{array}{l}< \\
0.001\end{array}$ \\
\hline No & $\begin{array}{l}51 \\
(68.9)\end{array}$ & $241(75.1)$ & & & $\begin{array}{l}51 \\
(68.9)\end{array}$ & $51(68.9)$ & & \\
\hline Yes & $\begin{array}{l}23 \\
(31.1)\end{array}$ & $80(24.9)$ & & & $\begin{array}{l}23 \\
(31.1)\end{array}$ & $23(31.1)$ & & \\
\hline \multirow{2}{*}{$\begin{array}{l}\text { Clavien-Dindo } \\
\text { classification } \\
\text { Grade I }\end{array}$} & & & 0.766 & 0.107 & & & 0.893 & 0.048 \\
\hline & $\begin{array}{l}6 \\
(8.1)\end{array}$ & $20(6.2)$ & & & $\begin{array}{l}6 \\
(8.1)\end{array}$ & $6(8.1)$ & & \\
\hline Grade II & $\begin{array}{l}9 \\
(12.2)\end{array}$ & $29(9.0)$ & & & $\begin{array}{l}9 \\
(12.2)\end{array}$ & $6(8.1)$ & & \\
\hline Grade III & $\begin{array}{l}6 \\
(8.1)\end{array}$ & $24(7.5)$ & & & $\begin{array}{l}6 \\
(8.1)\end{array}$ & $8(10.8)$ & & \\
\hline Grade IV & $\begin{array}{l}2 \\
(2.7)\end{array}$ & $7(2.2)$ & & & $\begin{array}{l}2 \\
(2.7)\end{array}$ & $3(4.1)$ & & \\
\hline $\begin{array}{l}\text { Postoperative } \\
\text { mortality }\end{array}$ & $0(0)$ & $0(0)$ & NA & & $0(0)$ & $0(0)$ & NA & \\
\hline \multicolumn{9}{|c|}{ Data are expressed as means \pm standard deviations or as $n(\%)$ unless otherwise specified. } \\
\hline \multicolumn{9}{|c|}{$\begin{array}{l}\text { *Conversion to laparoscopic or open surgery in the robotic group and conversion to open surgery in the } \\
\text { laparoscopic group for any reason. }\end{array}$} \\
\hline
\end{tabular}

The detailed complications are listed in eTable 1. The early and late complication rates were comparable between the groups. In early complications, local and systemic complication rates were also similar. Leakages after esophagojejunostomy were identified in three patients after robotic gastrectomy $(4.1 \%)$ and in seven patients after laparoscopic gastrectomy (2.2\%). The patients with esophagojejunostomy leakage underwent endoscopic and radiological interventions or reoperation.

\section{Textbook outcome}

Textbook outcomes were achieved in 70.3\% (50 of 74) in the RTG group and in 75.7\% (56 of 74) in the LTG group, which was not significantly different $(P=0.579)$. The distribution of each textbook outcome metric for both groups is presented in Fig. 1. Each quality metric was comparable between the two groups before and after matching. The quality metric that had the most negative impact on the proportion achieving textbook outcome was the absence of severe complications, which was achieved in only $77.0 \%$ in both groups (eTable 2). 


\section{Survival outcomes}

The median follow-up period was 51 months (interquartile range 26-83 months). Five (6.8\%) patients died in the RTG group, while $42(13.1 \%)$ patients died in the LTG group. In the entire cohort, there was no difference in the fiveyear overall survival rate between the RTG and LTG groups $(90.7 \%$ and $85.6 \%$, respectively; log-rank $P=0.082$; Fig. 2a). After matching, the five-year overall survival rate also did not differ between the RTG and LTG groups ( 90.7 and $85.9 \%$, respectively; log-rank $P=0.144$; Fig. 2b). The numbers of deaths or recurrences in the RTG and LTG groups were six (8.1\%) and 53 (16.5\%), respectively. In the entire cohort, five-year relapse-free survival rate was higher in the RTG group (89.5\%) than in the LTG group (82.6\%, log-rank $P=0.044$; Fig. 2c). However, after matching, five-year relapse-free survival rate was comparable between the RTG (89.5\%) and LTG (85.2\%) groups (log-rank $P=0.167$; Fig. 2d). Recurrence was recorded in two (2.7\%) patients in the RTG group and in 31 (9.7\%) patients in the LTG group; this difference was not statistically significant before and after matching $(P=0.061$ and $P=0.442$, respectively). Detailed recurrence patterns after RTG and LTG are listed in eTable 3.

\section{Prognostic factors for overall and relapse-free survival}

In univariate analysis, the prognostic factors associated with overall survival were age, BMI, the ASA class, tumour size, estimated blood loss, pathological TNM stage, and textbook outcome. The Cox regression model for multivariate analysis demonstrated that BMI, the ASA class, tumour size, pathological TNM stage, and textbook outcome were independent predictors of overall survival (Table 3). The prognostic factors for relapse-free survival in multivariate analysis were BMI, the ASA class, tumour size, pathological TNM stage, and textbook outcome (Table 4). Textbook outcome was an independent prognostic factor for both overall (hazard ratio [HR] 2.46, 95\% confidence interval $[\mathrm{CI}] 1.28-4.73 ; P=0.007)$ and relapse-free survival (HR 2.33, 95\% $\mathrm{Cl} 1.31-4.15, P=0.004)$. However, the operative method was not an independent predictor of overall $(P=0.299)$ and relapse-free survival $(P$ = 0.141). The HRs for overall and relapse-free survival in the RTG group compared with those in the LTG group were 0.60 (95\% Cl 0.23-1.57) and 0.52 (95\% Cl 0.21-1.25), respectively. 
Table 3

Univariate and multivariate Cox regression models for overall survival

\begin{tabular}{|c|c|c|c|c|c|}
\hline \multirow[b]{2}{*}{ Factors } & \multirow[b]{2}{*}{$\begin{array}{l}\text { Frequency } \\
(n=395)\end{array}$} & \multicolumn{2}{|l|}{ Univariate model } & \multicolumn{2}{|l|}{ Multivariate model } \\
\hline & & HR (95\%Cl) & $\mathrm{P}$ & $\mathrm{HR}(95 \% \mathrm{Cl})$ & $P$ \\
\hline Age, year ${ }^{a}$ & 395 & $1.63(1.25-2.23)$ & $<0.001$ & $1.24(0.94-1.64)$ & 0.136 \\
\hline \multicolumn{6}{|l|}{ Sex } \\
\hline Male & 265 & 1 & & & \\
\hline Female & 130 & $0.84(0.45-1.56)$ & 0.574 & & \\
\hline \multicolumn{6}{|c|}{ Body mass index, kg/m² } \\
\hline$<23$ & 151 & 1 & & 1 & \\
\hline$\geq 23$ & 244 & $0.46(0.26-0.81)$ & 0.008 & $0.48(0.26-0.89)$ & 0.019 \\
\hline \multicolumn{6}{|l|}{ ASA } \\
\hline 1 & 201 & 1 & & 1 & \\
\hline 2 & 184 & $2.18(1.16-4.09)$ & 0.016 & $1.69(0.81-3.53)$ & 0.163 \\
\hline 3 & 10 & $5.75(2.09-15.84)$ & 0.001 & $6.02(1.86-19.40)$ & 0.003 \\
\hline \multicolumn{6}{|l|}{ Tumor histology } \\
\hline Differentiated & 234 & 1 & & & \\
\hline Undifferentiated & 161 & $0.75(0.40-1.40)$ & 0.364 & & \\
\hline \multicolumn{6}{|l|}{ Tumor location } \\
\hline Upper third & 272 & 1 & & & \\
\hline Middle to lower third & 123 & $1.32(0.74-2.37)$ & 0.347 & & \\
\hline Tumor size, cm & 395 & $1.29(1.20-1.39)$ & $<0.001$ & $1.16(1.04-1.28)$ & 0.006 \\
\hline \multicolumn{6}{|l|}{ Operation method } \\
\hline Laparoscopy & 321 & 1 & & 1 & \\
\hline Robot & 74 & $0.45(0.18-1.14)$ & 0.091 & $0.60(0.23-1.57)$ & 0.299 \\
\hline Operation time, $\min { }^{b}$ & 395 & $1.04(0.99-1.10)$ & 0.119 & & \\
\hline
\end{tabular}

${ }^{a}$ the hazard ratio shown is for every 10 year increase in age.

$\mathrm{b}$ the hazard ratio shown is for every $10 \mathrm{~min}$ increase in operation time.

${ }^{c}$ the hazard ratio shown is for every $10 \mathrm{ml}$ increase in estimated blood loss.

$\mathrm{HR}$, hazard ratio; Cl, confidence interval; ASA, American Society of Anaesthesiologists. 


\begin{tabular}{|c|c|c|c|c|c|}
\hline & & Univariate model & & Multivariate model & \\
\hline Estimated blood loss, $\mathrm{ml}^{\mathrm{c}}$ & 395 & $1.02(1.01-1.03)$ & 0.003 & $1.001(0.99-1.02)$ & 0.882 \\
\hline \multicolumn{6}{|l|}{ Pathological TNM stage ${ }^{d}$} \\
\hline I & 257 & 1 & & 1 & \\
\hline II & 67 & $1.72(0.68-4.34)$ & 0.252 & $1.94(0.73-5.11)$ & 0.182 \\
\hline III & 71 & $7.75(4.14-14.50)$ & $<0.001$ & $4.21(1.86-9.53)$ & 0.001 \\
\hline \multicolumn{6}{|l|}{ Textbook outcome } \\
\hline Achieved & 243 & 1 & & 1 & \\
\hline Failed to achieve & 152 & $2.18(1.23-3.87)$ & 0.008 & $2.46(1.28-4.73)$ & 0.007 \\
\hline \multicolumn{6}{|c|}{ a the hazard ratio shown is for every 10 year increase in age. } \\
\hline \multicolumn{6}{|c|}{ b the hazard ratio shown is for every 10 min increase in operation time. } \\
\hline \multicolumn{6}{|c|}{${ }^{\mathrm{C}}$ the hazard ratio shown is for every $10 \mathrm{ml}$ increase in estimated blood loss. } \\
\hline \multicolumn{6}{|c|}{ HR, hazard ratio; $\mathrm{Cl}$, confidence interval; ASA, American Society of Anaesthesiologists. } \\
\hline
\end{tabular}


Table 4

Univariate and multivariate Cox regression models for relapse-free survival

\begin{tabular}{|c|c|c|c|c|c|}
\hline & & Univariate model & & Multivariate model & \\
\hline \multirow[t]{2}{*}{ Factors } & Frequency & $\mathrm{HR}(95 \% \mathrm{Cl})$ & $P$ & $\mathrm{HR}(95 \% \mathrm{Cl})$ & $P$ \\
\hline & $(n=395)$ & & & & \\
\hline Age, year a & 395 & $1.65(1.30-2.09)$ & $<0.001$ & $1.21(0.94-1.57)$ & 0.146 \\
\hline \multicolumn{6}{|l|}{ Sex } \\
\hline Male & 265 & 1 & & & \\
\hline Female & 130 & $0.80(0.45-1.40)$ & 0.427 & & \\
\hline \multicolumn{6}{|c|}{ Body mass index, $\mathrm{kg} / \mathrm{m}^{2}$} \\
\hline$<23$ & 151 & 1 & & 1 & \\
\hline$\geq 23$ & 244 & $0.51(0.30-0.84)$ & 0.009 & $0.56(0.33-0.96)$ & 0.034 \\
\hline \multicolumn{6}{|l|}{ ASA } \\
\hline 1 & 201 & 1 & & 1 & \\
\hline 2 & 184 & $1.86(1.08-3.22)$ & 0.026 & $1.43(0.75-2.71)$ & 0.277 \\
\hline 3 & 10 & $4.25(1.60-11.28)$ & 0.004 & $5.43(1.77-16.69)$ & 0.003 \\
\hline \multicolumn{6}{|l|}{ Tumor histology } \\
\hline Differentiated & 234 & 1 & & & \\
\hline Undifferentiated & 151 & $0.73(0.42-1.27)$ & 0.267 & & \\
\hline \multicolumn{6}{|l|}{ Tumor location } \\
\hline Upper third & 272 & 1 & & & \\
\hline Middle to lower third & 123 & $1.03(0.60-1.76)$ & 0.921 & & \\
\hline Tumor size, cm & 395 & $1.30(1.21-1.39)$ & $<0.001$ & $1.11(1.01-1.23)$ & 0.027 \\
\hline \multicolumn{6}{|l|}{ Operation method } \\
\hline Laparoscopy & 321 & 1 & & 1 & \\
\hline
\end{tabular}

\footnotetext{
a the hazard ratio shown is for every 10 year increase in age.

$\mathrm{b}$ the hazard ratio shown is for every 10 min increase in operation time.

${ }^{c}$ the hazard ratio shown is for every $10 \mathrm{ml}$ increase in estimated blood loss.

d Stage, according to the 8th edition of the American Joint Committee on Cancer staging system for gastric cancer.

$\mathrm{HR}$, hazard ratio; Cl, confidence interval; ASA, American Society of Anaesthesiologists.
} 


\begin{tabular}{|c|c|c|c|c|c|}
\hline & & Univariate model & & Multivariate model & \\
\hline Robot & 74 & $0.43(0.19-1.00)$ & 0.051 & $0.52(0.21-1.25)$ & 0.141 \\
\hline Operation time, $\min { }^{b}$ & 395 & $1.04(0.99-1.09)$ & 0.120 & & \\
\hline Estimated blood loss, $\mathrm{ml}^{\mathrm{c}}$ & 395 & $1.02(1.003-1.03)$ & 0.012 & $1.00(0.98-1.01)$ & 0.669 \\
\hline \multicolumn{6}{|l|}{ Pathological TNM stage ${ }^{d}$} \\
\hline I & 257 & 1 & & 1 & \\
\hline II & 67 & $2.14(0.93-4.93)$ & 0.075 & $2.30(0.95-5.56)$ & 0.064 \\
\hline III & 71 & $10.72(5.98-19.21)$ & $<0.001$ & $7.08(3.39-14.82)$ & 0.004 \\
\hline \multicolumn{6}{|l|}{ Textbook outcome } \\
\hline Achieved & 243 & 1 & & 1 & \\
\hline Failed to achieve & 152 & $2.03(1.22-3.38)$ & 0.007 & $2.33(1.31-4.15)$ & 0.004 \\
\hline \multicolumn{6}{|c|}{ a the hazard ratio shown is for every 10 year increase in age. } \\
\hline \multicolumn{6}{|c|}{${ }^{b}$ the hazard ratio shown is for every 10 min increase in operation time. } \\
\hline \multicolumn{6}{|c|}{ ' the hazard ratio shown is for every $10 \mathrm{ml}$ increase in estimated blood loss. } \\
\hline \multicolumn{6}{|c|}{$\begin{array}{l}\text { d Stage, according to the 8th edition of the American Joint Committee on Cancer staging system for gastric } \\
\text { cancer. }\end{array}$} \\
\hline
\end{tabular}

\section{Discussion}

We performed propensity score matched analysis to adjust for potential selection bias, and short- and long-term outcomes were similar between the groups following successful matching. The RTG group showed similar operative outcomes compared with the LTG group, except for a longer operative time. Textbook outcome as a composite measure was comparably achieved in both groups. Regarding the long-term outcomes of both groups, similar overall and relapse-free survival rates were observed. Textbook outcome was an independent predictor for survival; however, the operative method was not an independent prognostic factor of survival.

To date, most studies on short-term outcomes following minimally invasive total gastrectomy focused on single factors, such as bleeding, retrieved lymph nodes, hospital stay, complications, and mortality, to measure surgical quality[23]. Unlike standard surgical quality measures, textbook outcome integrates metrics for oncologically sound surgical treatment, and metrics for measuring clinical events (void of complications and prolonged admissions). Thus, textbook outcome is a helpful measure in evaluating the quality of gastrectomy[9]. To the best of our knowledge, this study is the first to evaluate the short-term outcomes of RTG compared with those of LTG for patients with gastric cancer using textbook outcome. 
In this study, textbook outcome was comparable between the RTG and LTG groups. Because robotic and laparoscopic surgeries fall into the same domain of minimally invasive approaches, it may be a major obstacle for robotic surgery to offer significant surgical advantages compared to laparoscopic surgery. Moreover, because RTG has been used for proximal gastric cancer since 2009 in our institution, RTG included initial and early experiences, while LTG included experiences after the learning curve in this study. Therefore, it would be more difficult to show the difference between the two methods in terms of textbook outcome.

Nevertheless, some studies have demonstrated that robotic gastrectomy has the advantage of lesser blood loss, higher number of retrieved lymph nodes, shorter hospital stay, and shorter learning curve than those of laparoscopic gastrectomy[14, 24, 25]. The number of retrieved lymph nodes and duration of hospital stay were included in the metrics of textbook outcome, while blood loss was not included in the quality metrics. In this study, blood loss tended to be lower in the RTG group than in the LTG group, although this was not statistically significant. Since intraoperative bleeding during gastrectomy is associated with the risk of tumour recurrence[2628], modifying the textbook outcome to include the metric for intraoperative blood loss may yield more valuable results in gastric cancer surgery.

In proximal gastric cancer, total gastrectomy with the minimally invasive approach including digestive tract reconstruction and splenic hilar lymphadenectomy is technically feasible; however, it is still a challenging procedure for many surgeons[29-31]. In addition, compared with distal gastrectomy, total gastrectomy inevitably causes some complications and is also a risk factor for postoperative morbidity and mortality[32]. Likewise, in this study, the overall complication rate was $31.1 \%$ in both groups of the matched cohort, which was also higher than the complication rate of distal gastrectomy in previous studies[3,5]. Further, the severe complication metric showed the most negative effect on the textbook outcome in both groups, while the other eight quality metrics were over $90 \%$ in both groups. Although minimally invasive total gastrectomy reduced postoperative complications compared with open total gastrectomy[33-35], a way to minimise complications after minimally invasive total gastrectomy should be considered.

Textbook outcome was achieved in $71.9 \%$ (284/395) of the study cohort, which was higher than the $32.1 \%$ reported in the Dutch Upper Gastrointestinal Cancer Audit group study[6]. The quality metric of "at least 15 lymph nodes examined" had the greatest negative effect on the textbook outcome in the Dutch study, while the severe complication metric showed the most negative effect on the textbook outcome in this study. The exclusion of palliative gastrectomy for survival analysis, relatively younger age of the patients, low ASA class, and earlier cancer stage of the study cohort may have contributed to higher achievement of the textbook outcome. As such, differences in patient characteristics and tumour biology would result in different achievement rates of each quality metric between this study and the Dutch study[36, 37].

In this study, overall and relapse-free survival rates were similar between both groups. In multivariable analysis, the operative method was not a prognostic factor for survival, whereas achieving textbook outcome was a prognostic factor for survival. Western studies also demonstrated that textbook outcome is strongly associated with longterm survival in gastric cancer surgery[8,9]. Therefore, the achievement of textbook outcome was significantly related to the improvement of survival rate, suggesting that the quality of surgery plays an important role in the survival of gastric cancer patients regardless of the operative method.

This study has the following limitations. First, this study had a small-scale, single-centre retrospective design. Therefore, we cannot exclude potential selection bias by the surgeon, although clinicopathological characteristics 
of the patients were well balanced between the groups after matching; of note, more advanced tumours were generally treated with laparoscopic surgery. Second, generalizability of our findings to the Western population is uncertain because this Eastern study cohort had relatively early gastric cancer with a small number of events. Large-scale randomised clinical trials will be able to confirm the results of our analysis. Finally, we did not evaluate functional long-term outcomes between the two groups. Thus, we could not compare the quality of life after minimally invasive total gastrectomy.

In conclusion, textbook outcome and survival after RTG for gastric cancer were comparable to those of LTG. RTG might be considered a minimally invasive surgical option for gastric cancer treatment, although the longer operation time remains a major concern. Achieving textbook outcome is strongly associated with improved longterm survival in patients undergoing total gastrectomy for gastric cancer.

\section{Declarations}

\section{Acknowledgements}

Not applicable

\section{Author Contributions}

CK Roh and SU Han designed and developed the protocol. S Lee, SY Son, and H Hur acquired the data. SY Son, H Hur, and SU Han contributed to the administrative, technical or material support. All authors wrote and reviewed the manuscript.

\section{Conflict of interest}

All authors have no conflicts of interest or financial ties to disclose.

\section{Ethics declarations}

The study was conducted in accordance with the "Declaration of Helsinki", and was approved by the institutional review board of Ajou University Hospital, Suwon, Korea (AJIRB-MED-MDB-19-144).

\section{References}

1 Landheer, M. L., Therasse, P. \& van de Velde, C. J. The importance of quality assurance in surgical oncology. Eur J Surg Oncol 28, 571-602, doi:10.1053/ejso.2002.1255 (2002).

2 Butterworth, J. W. et al. Challenges to quality assurance of surgical interventions in clinical oncology trials: A systematic review. Eur J Surg Oncol, doi:10.1016/j.ejso.2020.10.002 (2020).

3 Kim, W. et al. Decreased Morbidity of Laparoscopic Distal Gastrectomy Compared With Open Distal Gastrectomy for Stage I Gastric Cancer: Short-term Outcomes From a Multicenter Randomized Controlled Trial (KLASS-01). Annals of surgery 263, 28-35, doi:10.1097/SLA.0000000000001346 (2016).

4 Baiocchi, G. L. et al. International consensus on a complications list after gastrectomy for cancer. Gastric Cancer 22, 172-189, doi:10.1007/s10120-018-0839-5 (2019). 
5 Lee, H. J. et al. Short-term Outcomes of a Multicenter Randomized Controlled Trial Comparing Laparoscopic Distal Gastrectomy With D2 Lymphadenectomy to Open Distal Gastrectomy for Locally Advanced Gastric Cancer (KLASS-02-RCT). Annals of surgery 270, 983-991, doi:10.1097/SLA.0000000000003217 (2019).

6 Busweiler, L. A. et al. Textbook outcome as a composite measure in oesophagogastric cancer surgery. Br J Surg 104, 742-750, doi:10.1002/bjs.10486 (2017).

7 Salet, N. et al. Is Textbook Outcome a valuable composite measure for short-term outcomes of gastrointestinal treatments in the Netherlands using hospital information system data? A retrospective cohort study. BMJ Open 8, e019405, doi:10.1136/bmjopen-2017-019405 (2018).

8 van der Kaaij, R. T. et al. Using textbook outcome as a measure of quality of care in oesophagogastric cancer surgery. Br J Surg 105, 561-569, doi:10.1002/bjs.10729 (2018).

9 Levy, J. et al. Textbook Outcome and Survival in Patients With Gastric Cancer: An Analysis of the Population Registry of Esophageal and Stomach Tumours in Ontario (PRESTO). Annals of surgery, doi:10.1097/SLA.0000000000003849 (2020).

10 Kulshrestha, S. et al. Textbook oncologic outcome is associated with increased overall survival after esophagectomy. Surgery 168, 953-961, doi:10.1016/j.surg.2020.05.038 (2020).

11 Karthaus, E. G. et al. Textbook Outcome: A Composite Measure for Quality of Elective Aneurysm Surgery. Annals of surgery 266, 898-904, doi:10.1097/SLA.0000000000002388 (2017).

12 Ten Berge, M. G. et al. Textbook outcome as a composite outcome measure in non-small-cell lung cancer surgery. Eur J Cardiothorac Surg, doi:10.1093/ejcts/ezaa265 (2020).

13 van Roessel, S. et al. Textbook Outcome: Nationwide Analysis of a Novel Quality Measure in Pancreatic Surgery. Annals of surgery 271, 155-162, doi:10.1097/SLA.0000000000003451 (2020).

14 Uyama, I. et al. Clinical advantages of robotic gastrectomy for clinical stage I/II gastric cancer: a multiinstitutional prospective single-arm study. Gastric Cancer 22, 377-385, doi:10.1007/s10120-018-00906-8 (2019).

15 Liao, G., Zhao, Z., Khan, M., Yuan, Y. \& Li, X. Comparative analysis of robotic gastrectomy and laparoscopic gastrectomy for gastric cancer in terms of their long-term oncological outcomes: a meta-analysis of 3410 gastric cancer patients. World J Surg Oncol 17, 86, doi:10.1186/s12957-019-1628-2 (2019).

16 Roh, C. K. et al. Comparison of surgical outcomes between integrated robotic and conventional laparoscopic surgery for distal gastrectomy: a propensity score matching analysis. Sci Rep 10, 485, doi:10.1038/s41598-020-57413-z (2020).

17 Seo, W. J. et al. Reduced-port totally robotic distal subtotal gastrectomy for gastric cancer: 100 consecutive cases in comparison with conventional robotic and laparoscopic distal subtotal gastrectomy. Sci Rep 10, 16015, doi:10.1038/s41598-020-73118-9 (2020).

18 Shin, H. J. et al. Long-term Comparison of Robotic and Laparoscopic Gastrectomy for Gastric Cancer: A Propensity Score-weighted Analysis of 2084 Consecutive Patients. Annals of surgery, doi:10.1097/SLA.0000000000003845 (2020). 
19 Lu, J. et al. Assessment of Robotic Versus Laparoscopic Distal Gastrectomy for Gastric Cancer: A Randomized Controlled Trial. Annals of surgery, doi:10.1097/SLA.0000000000004466 (2020).

20 Kang, B. H. et al. Comparison of Surgical Outcomes between Robotic and Laparoscopic Gastrectomy for Gastric Cancer: The Learning Curve of Robotic Surgery. J Gastric Cancer 12, 156-163, doi:10.5230/jgc.2012.12.3.156 (2012).

21 Japanese Gastric Cancer, A. Japanese gastric cancer treatment guidelines 2014 (ver. 4). Gastric Cancer 20, 1-19, doi:10.1007/s10120-016-0622-4 (2017).

22 Guideline Committee of the Korean Gastric Cancer A. Korean Practice Guideline for Gastric Cancer 2018: an Evidence-based, Multi-disciplinary Approach. J Gastric Cancer 19, 1-48, doi:10.5230/jgc.2019.19.e8 (2019).

23 Shibasaki, S., Suda, K., Obama, K., Yoshida, M. \& Uyama, I. Should robotic gastrectomy become a standard surgical treatment option for gastric cancer? Surg Today 50, 955-965, doi:10.1007/s00595-019-01875-w (2020).

24 Wang, W. J. et al. Severity and incidence of complications assessed by the Clavien-Dindo classification following robotic and laparoscopic gastrectomy for advanced gastric cancer: a retrospective and propensity scorematched study. Surg Endosc 33, 3341-3354, doi:10.1007/s00464-018-06624-7 (2019).

25 Suda, K. et al. Potential advantages of robotic radical gastrectomy for gastric adenocarcinoma in comparison with conventional laparoscopic approach: a single institutional retrospective comparative cohort study. Surg Endosc 29, 673-685, doi:10.1007/s00464-014-3718-0 (2015).

26 Kamei, T., Kitayama, J., Yamashita, H. \& Nagawa, H. Intraoperative blood loss is a critical risk factor for peritoneal recurrence after curative resection of advanced gastric cancer. World J Surg 33, 1240-1246, doi:10.1007/s00268-009-9979-4 (2009).

27 Liang, Y. X. et al. Impact of intraoperative blood loss on survival after curative resection for gastric cancer. World J Gastroenterol 19, 5542-5550, doi:10.3748/wjg.v19.i33.5542 (2013).

28 Arita, T. et al. Increase in peritoneal recurrence induced by intraoperative hemorrhage in gastrectomy. Ann Surg Oncol 22, 758-764, doi:10.1245/s10434-014-4060-4 (2015).

29 Kodera, Y. et al. Introducing laparoscopic total gastrectomy for gastric cancer in general practice: a retrospective cohort study based on a nationwide registry database in Japan. Gastric Cancer 22, 202-213, doi:10.1007/s10120-018-0795-0 (2019).

30 Hyung, W. J. et al. A feasibility study of laparoscopic total gastrectomy for clinical stage I gastric cancer: a prospective multi-center phase II clinical trial, KLASS 03. Gastric Cancer 22, 214-222, doi:10.1007/s10120-0180864-4 (2019).

31 Yang, K. et al. Robotic spleen-preserving splenic hilar lymph node dissection during total gastrectomy for gastric cancer. Surg Endosc 33, 2357-2363, doi:10.1007/s00464-019-06772-4 (2019).

32 Lee, K. G. et al. Risk factors associated with complication following gastrectomy for gastric cancer: retrospective analysis of prospectively collected data based on the Clavien-Dindo system. J Gastrointest Surg 18, 1269-1277, doi:10.1007/s11605-014-2525-1 (2014).

Page 20/23 
33 Haverkamp, L. et al. Laparoscopic total gastrectomy versus open total gastrectomy for cancer: a systematic review and meta-analysis. Surg Endosc 27, 1509-1520, doi:10.1007/s00464-012-2661-1 (2013).

34 Sweigert, P. J. et al. Total gastrectomy in patients with gastric adenocarcinoma: Is there an advantage to the minimally invasive approach? Surgery 166, 623-631, doi:10.1016/j.surg.2019.05.041 (2019).

35 Sakamoto, T., Fujiogi, M., Matsui, H., Fushimi, K. \& Yasunaga, H. Short-Term Outcomes of Laparoscopic and Open Total Gastrectomy for Gastric Cancer: A Nationwide Retrospective Cohort Analysis. Ann Surg Oncol 27, 518526, doi:10.1245/s10434-019-07688-y (2020).

36 Yamada, T. et al. The survival difference between gastric cancer patients from the UK and Japan remains after weighted propensity score analysis considering all background factors. Gastric Cancer 19, 479-489, doi:10.1007/s10120-015-0480-5 (2016).

37 van der Wielen, N., Straatman, J., Cuesta, M. A., Daams, F. \& van der Peet, D. L. Short-term outcomes in minimally invasive versus open gastrectomy: the differences between East and West. A systematic review of the literature. Gastric Cancer 21, 19-30, doi:10.1007/s10120-017-0747-0 (2018).

\section{Figures}


A

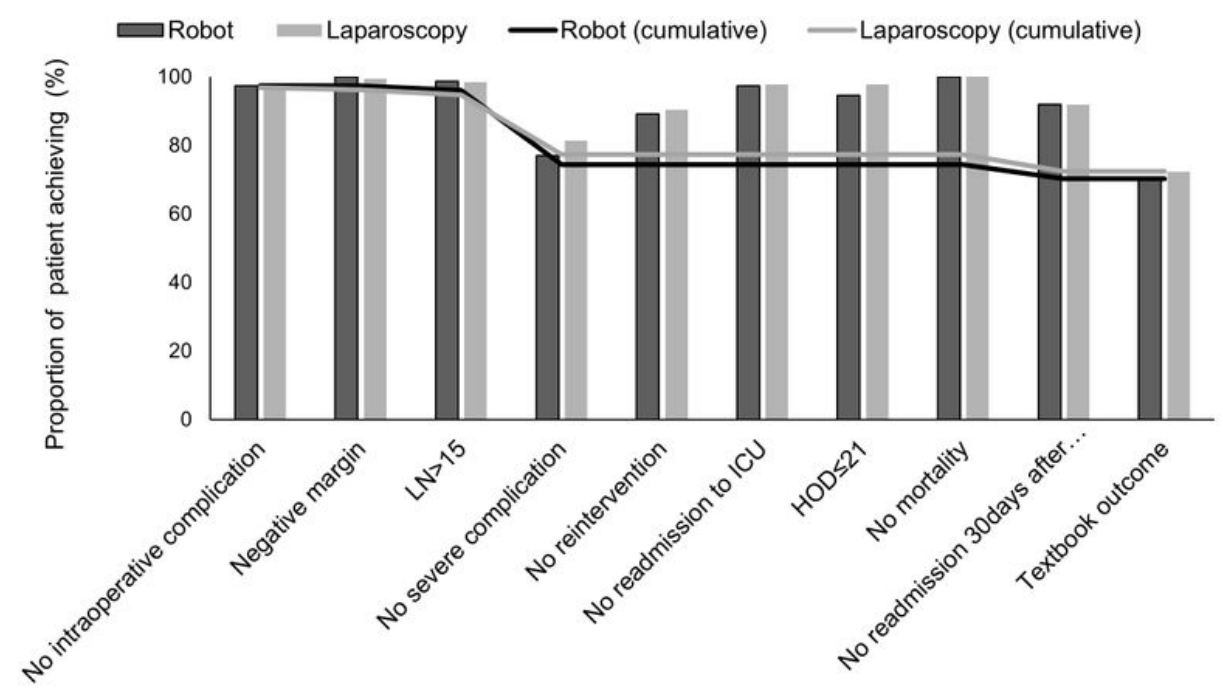

B

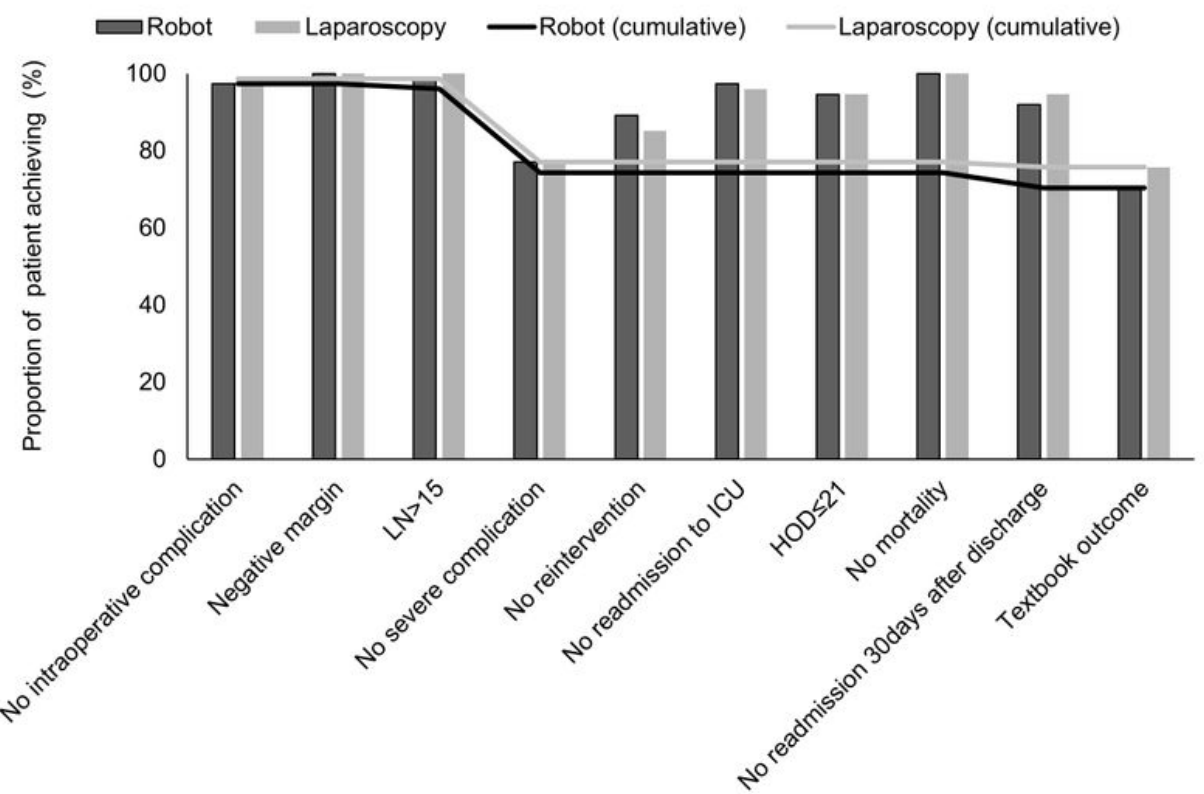

Figure 1

Proportion of patients achieving textbook outcome and each quality metric A. Entire cohort B. Matched cohort LN, lymph node; ICU, intensive care unit; HOD, hospital days. 

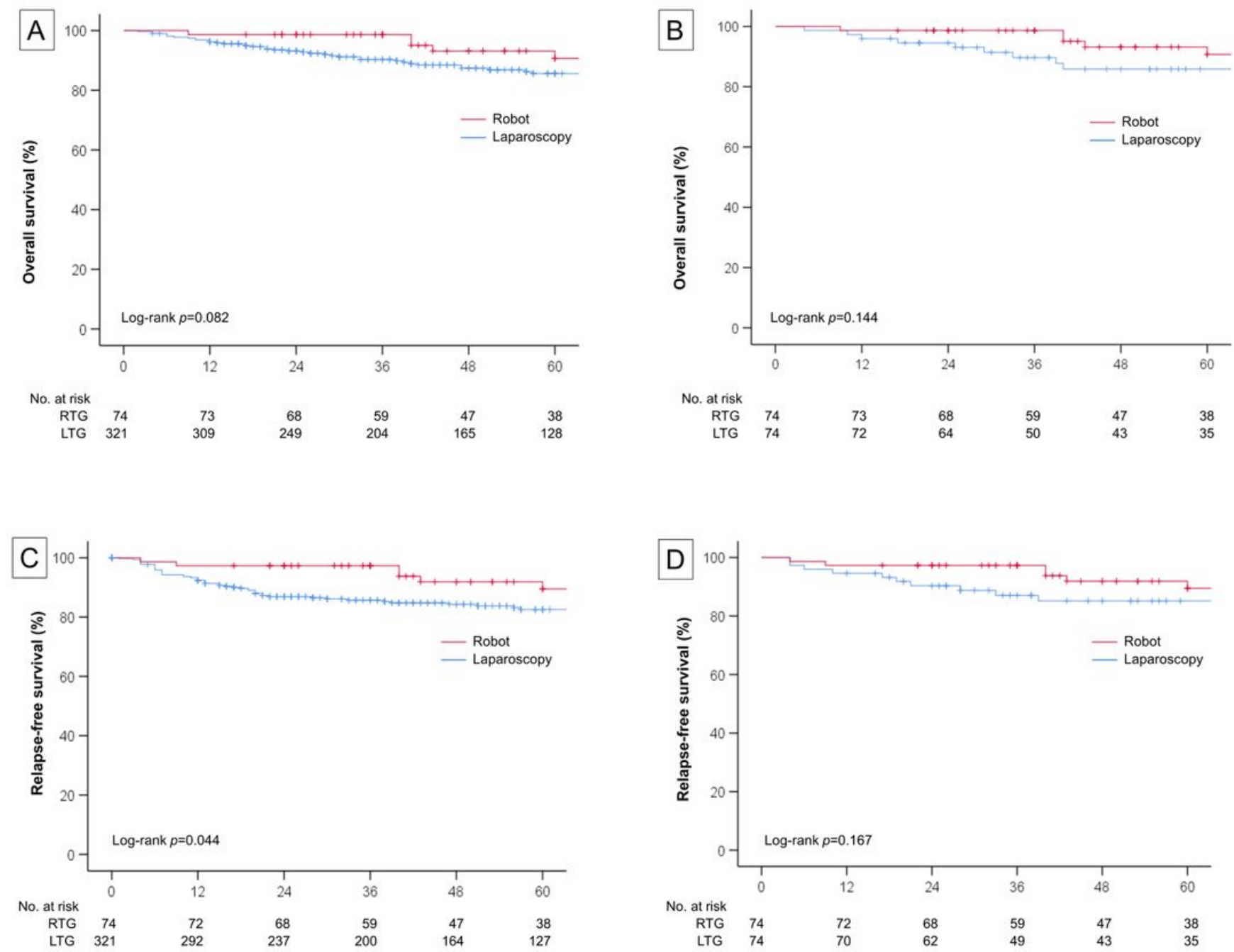

\section{Figure 2}

Kaplan-Meier curves for five-year overall and relapse-free survival A. Five-year overall survival of the entire cohort B. Five-year overall survival of the matched cohort C. Five-year relapse-free survival of the entire cohort D. Five-year relapse-free survival of the matched cohort RTG, robotic total gastrectomy; LTG, laparoscopic total gastrectomy

\section{Supplementary Files}

This is a list of supplementary files associated with this preprint. Click to download.

- SupplementarySR.pdf 